\title{
A Fast Chemometrics Approach to Quantitative Analysis of Metformin Hydrochloride, Enalapril Maleate, and Captopril in Tablets Based on HPLC-PAD Spectra
}

\author{
Jules Muhire, Bao Qiong Li, Hong Lin Zhai", Xue Wang and Min Li Xu \\ College of Chemistry \& Chemical Engineering, Lanzhou University, Lanzhou, 730000, PR China
}

Received: 02 May 2018; accepted: 04 May 2018

\begin{abstract}
Diabetes mellitus and concurrent hypertension disorder are dreadful all over the world and are often managed by some drugs, such as metformin hydrochloride (MFH), enalapril maleate (ENM), and captopril (CAP). In this work, a reliable and fast quantitative analysis of these three components in tablets was carried out by Tchebichef image moment method and multivariate curve resolution with alternating least squares on three-dimensional (3D) spectra obtained by high-performance liquid chromatography coupled with photodiode array detection (HPLC-PAD). 3D spectra were obtained within only $2 \mathrm{~min}$, and linear quantitative models were established by stepwise regression based on the calculated image moments. Among these two methods, Tchebichef image moment method showed outcome distinction. The correlation coefficients of cross-validation $\left(R_{\text {Loo-cv }}\right)$ are more than 0.988 , while their recoveries are $100.1 \pm 1.7 \%$ $(\mathrm{MFH}), 95.4 \pm 5.4 \%(\mathrm{ENM})$, and $105.3 \pm 5.7 \%(\mathrm{CAP})$, respectively. The intra- and inter-day precisions $(R S D)$ are less than $5.42 \%$. The proposed methods were also applied to the analysis of real tablets. This study reveals the effectiveness and convenience of the proposed image-moment method that may be a potential technology for the quality control and investigation of drugs in routine analysis.
\end{abstract}

Keywords: HPLC-PAD, Tchebichef moments, quantitative analysis, metformin hydrochloride, enalapril maleate, captopril

\section{Introduction}

Diabetes mellitus is a chronic metabolic disorder characterized by hyperglycaemia, in which these two are the most common diseases of this era, and statistically, over 300 million people are affected by diabetes globally [1]. Metformin hydrochloride (MFH), a developed drug from a herb, Galega officinalis, has been used as the foremost line therapy in the treatment of type 2 diabetes mellitus patients [2]. Chemically, it is $N, N$-dimethylimidocarbonimidic diamide hydrochloride [3] and has the structural formula as indicated in Figure 1A. As demonstrated in the literature [4], $500 \mathrm{mg}$ MFH should be taken twice or thrice a day by adult people, whereas children under the age of 18 are not allowed to take it.

For many patients with type 2 diabetes, monotherapy with an oral anti-diabetic agent is not sufficient to attain glycaemic target, and some drugs may be required to fulfil a suitable control. Thirty percent of patients with type 2 diabetes have hypertension at the time of diagnosis of diabetes [5]. Fortunately, it has been found that the angiotensin-converting enzyme (ACE) inhibitors are pharmaceutical drugs, either separately or in combination with other medications [6]. As ACE inhibitors are mostly used for the treatment of hypertension and heart failure, enalapril maleate (ENM) [7] and captopril (CAP) [8] are often taken with MFH (the chemical structures are also shown in Figures 1B and 1C). $50 \mathrm{mg}$ CAP is prescribed for once-daily formulation, whereas $10 \mathrm{mg}$ ENM is for once- or twice-daily dosing [9]. Although the analysis of three components have been reported [6c, 10], a more rapid, simple, and precise quantitative analysis of these drugs is very important for clinical medication safety.

*Author for correspondence: zhaihl@163.com; tel.: +86 931 8912596; fax: +869318912582.
High-performance liquid chromatography coupled with photodiode-array detection (HPLC-PAD) has been broadly used in various analytical domains due to its abundant information represented with a three-dimensional (3D) spectrum [11]. However, for further analysis of complex samples, it is time-consuming due to the pre-treatments of samples and the optimization of experimental conditions [6a, 10c, 12]. Furthermore, several interferences, such as overlapping and shifts of peaks, baseline drifting, and noise, cause the difficulty of accurate analysis [6c, 13]. Favourably, chemometric methods can provide the solutions to overcome such main challenges.

Image moments are frequently used as descriptors in image analysis. The invariant of such moments is a vital characteristic, which means that image moments remain unchanged during distortions like translation, scale, and rotation, and the powerful multi-resolution capability is another significant property. In addition, the moments with discrete and orthogonal properties are usually preferable due to its less information redundancy and do not have computational approximations as well [14]. Discrete orthogonal moments include Hahn moments, Krawtchouk moments, and Tchebichef moments [15], and they played an important role in the data analysis by extracting more suitable information. Besides, among these moments, Tchebichef moments (TMs) demonstrated superiority to other discrete moment methods, since Tchebichef polynomials have a unit weight function which makes it an ideal candidate for extracting global features of the images [16]. Image moments had contributed in various analytical techniques for handling the challenges found in their spectral analysis, for instance, in fluorescence three-dimensional spectra of different drugs [17], LC-MS [18], and multi-set ${ }^{1} \mathrm{H}$ nuclear magnetic resonance [19].

A classical method, multivariate curve resolution optimized by alternating least squares (MCR-ALS), had been also significantly applied to different fields of analytical techniques, such as the

This is an open-access article distributed under the terms of the Creative Commons Attribution-NonCommercial 4.0 International License (https://creativecommons.org/licenses/by-nc/4.0/), which permits unrestricted use, distribution, and reproduction in any medium for non-commercial purposes, provided the original author and source are credited, a link to the CC License is provided, and changes - if any - are indicated. 
<smiles>C[C@H](CS)C(=O)N1CCC[C@H]1C(=O)O</smiles><smiles>C=C([C@@H](C)N[C@@H](CCc1ccccc1)C(=O)OCC)N1CCC[C@H]1C(=O)O</smiles>

Figure 1. Chemical structures: (A) metformin hydrochloride, (B) enalapril maleate, and (C) captopril

near and mid-infrared spectroscopies by modelling the protein process [20], the analysis of spectroscopic data of chemical reactions [21], and the liquid/gas chromatography coupled with mass spectrometry [22]. In addition, this approach, as a three-way resolution procedure, has been utilized to analytically resolve the signal shift, shape distortions, and peak overlaps in chromatographic systems [23]. Nevertheless, the comparison of this method with any other reliable modelling and predicting method is highly desirable.

Therefore, Tchebichef image moment (TM) method was proposed and compared with MCR-ALS in the prediction and quantitative analysis of the spectra of MFH, ENM, and CAP in tablets which are simultaneously determined by HPLC-PAD for the first time.

\section{Experimental}

2.1. Chemicals and Reagents. Metformin hydrochloride (MFH, CAS: 1115-70-4), enalapril maleate (ENM, CAS: 76095-16-4), and captopril (CAP, CAS: 62571-86-2) reference standards were purchased from Aladdin Industrial Corporation (Shanghai, China). The tablets were bought from a nearby market. Analytical-grade ortho-phosphoric acid and HPLC-grade methanol were purchased from Biaoshiqi (Tianjin, China). All other used chemicals were of analytical grade. High-purity water was purified using a vacuum pump purification system prior to the preparation of mobile phase.

2.2. Preparation of Solutions. MFH, ENM, and CAP standards were weighed precisely, well mixed by uniform design $\left(U_{25}^{*}\left(25^{11}\right)\right)$, and then transferred to $10 \mathrm{~mL}$ volumetric flasks as the standard stock solutions. Then, some volume of the standard stock solutions was transferred to a $10 \mathrm{~mL}$ volumetric flask and diluted with a mixed solution of methanol and water $(3: 2, v / v)$ to prepare working solutions (calibration standard samples) at twenty-five levels. Their concentrations are listed in Table 1 in the Exp. column. The tablets were accurately weighed, ground into powder, dissolved in HPLC-grade methanol, and centrifuged. Then, the supernatant was obtained.
The mobile phase consisted of methanol $(80 \%, v)$ and ultrapure water $(20 \%, v)$, and the $\mathrm{pH}$ was adjusted to 3.28 by using dilute ortho-phosphoric acid with a calibrated digital $\mathrm{pH}$ meter.

All of solvents were filtered through a $0.22 \mu \mathrm{m}$ microfiltration membrane (made in Shanghai, China).

2.3. Instrumentation and Chromatographic Conditions. A Waters 1525 binary HPLC pump system coupled with photodiode array detector was adopted, and the data acquisition was performed by Breeze 2 software. The operation was completed on the Waters column (Symmetry ${ }^{\circledR}$ C18 $3.5 \mu \mathrm{m}$ particle size; $4.6 \mathrm{~mm} \times 75 \mathrm{~mm}$ ) with a constant flow rate of $1.0 \mathrm{~mL} / \mathrm{min}$. The injection volume was $5 \mu \mathrm{L}$ for both standard and real samples. All injections were done at room temperature. The detection wavelength was in the range of $200-260 \mathrm{~nm}$.

\subsection{Analytical Method}

2.4.1. Spectrum and Its Grayscale Image. A 3D spectrum obtained from HPLC-PAD was exported as a 2D matrix, with a column retention time (ranging from 0.0 to $3.5 \mathrm{~min}$ ) and a row wavelength (ranging from 200 to $260 \mathrm{~nm}$ ), in which the 2D matrix was represented by its grayscale image by keeping the same storage format.

2.4.2. Calculation of Tchebichef Moments. Tchebichef moments include in the discrete orthogonal moments, where Tchebichef polynomials fulfil the orthogonal property in the discrete domain of molecular image coordinate space, and the computation of these Tchebichef moments does not necessitate any discrete approximation [24].

For a grayscale image $f(x, y)$ with the size of $N \times M$, the Tchebichef moments (TMs) with the order $(n+m)$ are defined as follows [25]:

$$
\begin{aligned}
& T_{n, m}=\frac{1}{\rho(n, N) \rho(m, M)} \sum_{x=0}^{N-1} \sum_{y=0}^{M-1} t_{n}(x) t_{m}(y) f(x, y) \\
& (n=0,1,2, \ldots N-1, m=0,1,2, \ldots M-1) .
\end{aligned}
$$


Table 1. The experimental and calculated concentrations $(\mu \mathrm{g} / \mathrm{mL})$ of the three target components for calibration and test sets

\begin{tabular}{|c|c|c|c|c|c|c|c|c|c|}
\hline \multirow[t]{3}{*}{ Samples } & \multicolumn{3}{|c|}{ Metformin hydrochloride } & \multicolumn{3}{|c|}{ Enalapril maleate } & \multicolumn{3}{|c|}{ Captopril } \\
\hline & \multirow[t]{2}{*}{ Exp. } & \multicolumn{2}{|c|}{ Cal. } & \multirow[t]{2}{*}{ Exp. } & \multicolumn{2}{|c|}{ Cal. } & \multirow[t]{2}{*}{ Exp. } & \multicolumn{2}{|c|}{ Cal. } \\
\hline & & $\mathrm{TM}$ & MCR-ALS & & TM & MCR-ALS & & TM & MCR-ALS \\
\hline 1 & 160.0 & 165.2 & 109.6 & 224.0 & 222.3 & 165.1 & 876.0 & 891.2 & 1154.5 \\
\hline 2 & 114.0 & 108.4 & 121.1 & 166.0 & 166.4 & 187.7 & 1200 & 1193 & 1084.9 \\
\hline 3 & 180.0 & 178.9 & 151.2 & 250.0 & 248.1 & 235.6 & 864.0 & 854.2 & 1085.3 \\
\hline 4 & 140.0 & 135.9 & 179.8 & 188.0 & 191.3 & 274.9 & 890.0 & 885.6 & 1083.3 \\
\hline 5 & 224.0 & 224.8 & 172.4 & 272.0 & 277.8 & 298.3 & 500.0 & 507.4 & 1112.3 \\
\hline 7 & 122.0 & 121.1 & 195.6 & 200.0 & 205.4 & 203.8 & 1150 & 1168 & 915.8 \\
\hline 8 & 132.0 & 130.5 & 238.9 & 280.0 & 283.0 & 230.8 & 700.0 & 687.8 & 928.7 \\
\hline $9^{p}$ & 164.0 & 181.3 & 102.0 & 176.0 & 176.2 & 246.6 & 600.0 & 588.7 & 836.5 \\
\hline $10^{p}$ & 240.0 & 239.9 & 119.6 & 184.0 & 159.2 & 274.7 & 800.0 & 792.0 & 838.5 \\
\hline 11 & 110.0 & 114.6 & 129.9 & 218.0 & 219.6 & 184.2 & 760.0 & 752.7 & 917.8 \\
\hline $12^{p}$ & 120.0 & 119.7 & 136.4 & 248.0 & 257.2 & 198.8 & 740.0 & 740.2 & 883.6 \\
\hline $13^{p}$ & 204.0 & 213.7 & 164.5 & 240.0 & 248.4 & 234.2 & 550.0 & 529.3 & 924.2 \\
\hline 14 & 174.0 & 167.9 & 189.7 & 300.0 & 295.6 & 265.7 & 1040 & 1043 & 924.0 \\
\hline 17 & 200.0 & 193.5 & 233.3 & 284.0 & 285.3 & 199.1 & 850.0 & 861.0 & 827.3 \\
\hline $18^{p}$ & 150.0 & 161.0 & 112.2 & 140.0 & 134.2 & 192.5 & 660.0 & 593.6 & 771.6 \\
\hline 19 & 128.0 & 130.4 & 118.3 & 152.0 & 160.4 & 227.7 & 900.0 & 906.1 & 725.6 \\
\hline $20^{p}$ & 236.0 & 246.6 & 134.1 & 230.0 & 214.5 & 256.3 & 960.0 & 946.4 & 692.9 \\
\hline 21 & 184.0 & 187.2 & 142.2 & 208.0 & 205.2 & 138.4 & 560.0 & 578.3 & 641.0 \\
\hline 22 & 138.0 & 138.7 & 166.4 & 232.0 & 228.4 & 185.4 & 1100 & 1104 & 608.1 \\
\hline 23 & 196.0 & 203.2 & 168.0 & 160.0 & 164.9 & 189.5 & 1000 & 994.1 & 531.6 \\
\hline $24^{p}$ & 100.0 & 109.0 & 203.4 & 256.0 & 260.0 & 206.8 & 940.0 & 890.2 & 568.4 \\
\hline 25 & 116.0 & 117.9 & 225.7 & 290.0 & 282.8 & 256.9 & 930.0 & 918.6 & 513.6 \\
\hline
\end{tabular}

Equation 1 illustrates that the Tchebichef moments are defined directly in the discrete domain of the image $(f(x, y))$.

The most important property of set $t_{n}(x)$ is its orthogonality in the discrete domain:

$$
\sum_{x=0}^{N-1} t_{m}(x) t_{n}(x)=\rho(n, N) \delta_{n m}(0 \leq n \leq N-1,0 \leq m \leq M-1)
$$

where $\delta_{n m}$ is the Kronecker symbol, $\rho(n, N)$ is the squarenorm, and $t_{n}(x)$ is the discrete Tchebichef polynomials with order $n$ having the following recurrence relation:

$$
\begin{aligned}
& \begin{aligned}
&(n+1) t_{n+1}(x)-(2 n+1)(2 x-N+1) t_{n}(x) \\
&+n\left(N^{2}-n^{2}\right) t_{n-1}(x)=0, \\
& n=1,2, \ldots N-1 .
\end{aligned}
\end{aligned}
$$

With the aim to get a numerical stability as well as limited dynamical range, the normalized Tchebichef polynomials were established:

$$
\widetilde{t}_{n}(x)=\frac{t_{n}(x)}{\beta(n, N)}, \beta(n, N)=N^{n r}
$$

where the $\beta(n, N)$ stands for the suitable constant that is independent of $x$. Then the squared-norm of the normalized polynomials can be modified as follows:

$$
\widetilde{\rho}(n, N)=\frac{\rho(n, N)}{\beta^{2}(n, N)}
$$

Mukundan [26] proposed normalizing the Tchebichef polynomials by this $\beta(n, N)=N^{n}$, which is the simplest and suitable constant; Tchebichef moments can then be defined as:

$$
\begin{aligned}
& T_{n, m}=\frac{1}{\widetilde{\rho}(n, N) \widetilde{\rho}(m, M)} \sum_{x=0}^{N-1} \sum_{y=0}^{M-1} \widetilde{t}_{n}(x) \widetilde{t}_{m}(y) f(x, y) \\
& (n=0,1,2, \ldots N-1, m=0,1,2, \ldots M-1) .
\end{aligned}
$$

In this article, the following normalized recursive algorithm was used to accelerate the computation of Tchebichef polynomials [27]:

$$
\begin{aligned}
\widetilde{t}_{n}(x)= & \frac{(2 n-1)(2 x-N+1) \widetilde{t}_{n-1}(x)}{n N} \\
& -\frac{(n-1)\left[N^{2}-(n-1)^{2}\right] \widetilde{t}_{n-2}(x)}{n N^{2}}, \\
\widetilde{t}_{0}(x)= & 1 \\
\widetilde{t}_{1}(x)= & \frac{2 x+1-N}{N}, \\
\widetilde{\rho}(n) & =\left(\frac{2 n-1}{2 n+1}\right)\left(1-\frac{n^{2}}{N^{2}}\right) \widetilde{\rho}(n-1), \\
\widetilde{\rho}(0) & =N .
\end{aligned}
$$

The discrete orthogonal polynomials are always employed as the basic function in image reconstruction kernel, and the general image reconstruction function is as follows:

$$
\hat{f}(x, y)=\sum_{n=0}^{n N} \sum_{m=0}^{m M} T_{n, m} \widetilde{t}_{n}(x) \widetilde{t}_{m}(y)
$$

where $f(x, y)$ is the intensity function of the reconstructed image and $n N$ and $m M$ are the selected maximum orders $(n N<N-1, m M<M-1)$ for $n$ and $m$, respectively. The reconstruction error $\varepsilon$ (the difference of the original image and reconstructed one) can be used to the selection of 
optimal order $n N$ and $m M$ and calculated by the following formula.

$$
\varepsilon=\sum_{x=0}^{N-1} \sum_{y=0}^{M-1}|f(x, y)-\hat{f}(x, y)|
$$

2.5. Validation of Linear Models. By means of stepwise regression [18], the linear quantitative models for MFH, ENM, and CAP were established in accordance with the formula $Y=$ $A \times X+B$, in which $Y$ represents the concentrations of each target compound $(n=25)$ in mixed standard solutions $\left(\mu \mathrm{gL}^{-1}\right)$ and $X$ represents the Tchebichef moments selected by stepwise regression. To evaluate the performance of the established models, statistical parameters are calculated, such as the correlation coefficients $(R)$, adjusted correlation coefficients $\left(R_{\text {adj }}\right)$ [28], leave-one-out correlation coefficient $\left(R_{\mathrm{LOO}-\mathrm{cv}}\right)$, root mean square errors (RMSES), RMSE $E_{\mathrm{cv}}$ for cross-validation, $F$-test, and $p$-value. According to a study [29], the method performance is validated by the analytical precision and accuracy, including intra- and inter-day precisions (relative standard deviation [RSD]) and recovery,

$$
\text { Recovery }(\%)=\frac{\text { found amount }- \text { original amount }}{\text { added amount }} \times 100
$$

The whole calculation programs were written in M-file based on MATLAB 7.0 and accomplished well on a PC (CPU 3.40 GHz, RAM 16.0 GB).

\section{Results and Discussion}

3.1. HPLC-PAD Analysis and Tchebichef Moments. As can be seen in Figure 2, the shifts of peaks in retention time are the common phenomena due to minor fluctuations in the experimental conditions, which must be aligned to eliminate error in traditional chemometrics methods. Owing to the advantage of the inherently invariance property in translation, rotation, and scaling of images, the calculated TMs could not be affected by the shifts of peaks. Moreover, the TMs with different orders $(n, m)$ could decompose the shape information due to its powerful multi-resolution capability, which is beneficial to obtain the features of target components in overlapping information. Therefore, the requirement of baseline separation in traditional chromatographic analysis is no longer needed, which not only reduces the determination time but also simplifies the experimental operations. As a result, our determination was carried out within $2 \mathrm{~min}$, which is shorter than that of in the literature [10c, 12a], and the further analysis was done by the proposed method.

3.2. Linear Models. In order to handle irrelevant information and obtain more accurate results, two segments were selected in each grayscale image of $3 \mathrm{D}$ spectra according to the signals of target components as shown in Figure 3. Region I exhibits MFH, and region II includes ENM and CAP (partial overlapping). The TMs of the regions I and II were calculated by Eq. 6 .

It is known that high order moments are more sensitive to noise than low ones [30], i.e., the noise is localized in high frequencies. According to the change of reconstruction errors (Eqs. 8 and 9), the maximum orders of TMs were determined as $n N$ of 39 and $m M$ of 15 , which produced a total of $640[(n N+1)(m M+1)]$ image moments with different orders for each part. Then, the linear quantitative models for MFH, ENM, and CAP were established by stepwise regression, in which the concentrations of the three target components were regarded as the dependent variables $(C)$, while the calculated TMs were denoted as independent variables $\left(T_{n, m}\right)$.

Model 1 for MFH:

$$
\begin{aligned}
C_{\mathrm{MFH}}= & 82.41-3.597 \times 10^{3} \times T_{0,14}+9.375 \times 10^{3} \times T_{30,10} \\
& -9.842 \times 10^{3} \times T_{36,14}
\end{aligned}
$$

Model 2 for ENM:

$$
\begin{aligned}
C_{\mathrm{ENM}}= & 38.25+9.466 \times 10^{3} \times T_{21,3}-1.765 \times 10^{5} \times T_{29,3} \\
& -1.518 \times 10^{5} \times T_{38,7}+1.451 \times 10^{5} \times T_{39,14}
\end{aligned}
$$

Model 3 for CAP:

$$
\begin{aligned}
C_{\mathrm{CAP}}= & 487.5-1.429 \times 10^{3} \times T_{3,9}-1.574 \times 10^{3} \times T_{9,10} \\
& -1.004 \times 10^{6} \times T_{32,4}-6.692 \times 10^{5} \times T_{35,0}-4.669 \\
& \times 10^{5} \times T_{36,14}-4.491 \times 10^{5} \times T_{37,6}
\end{aligned}
$$

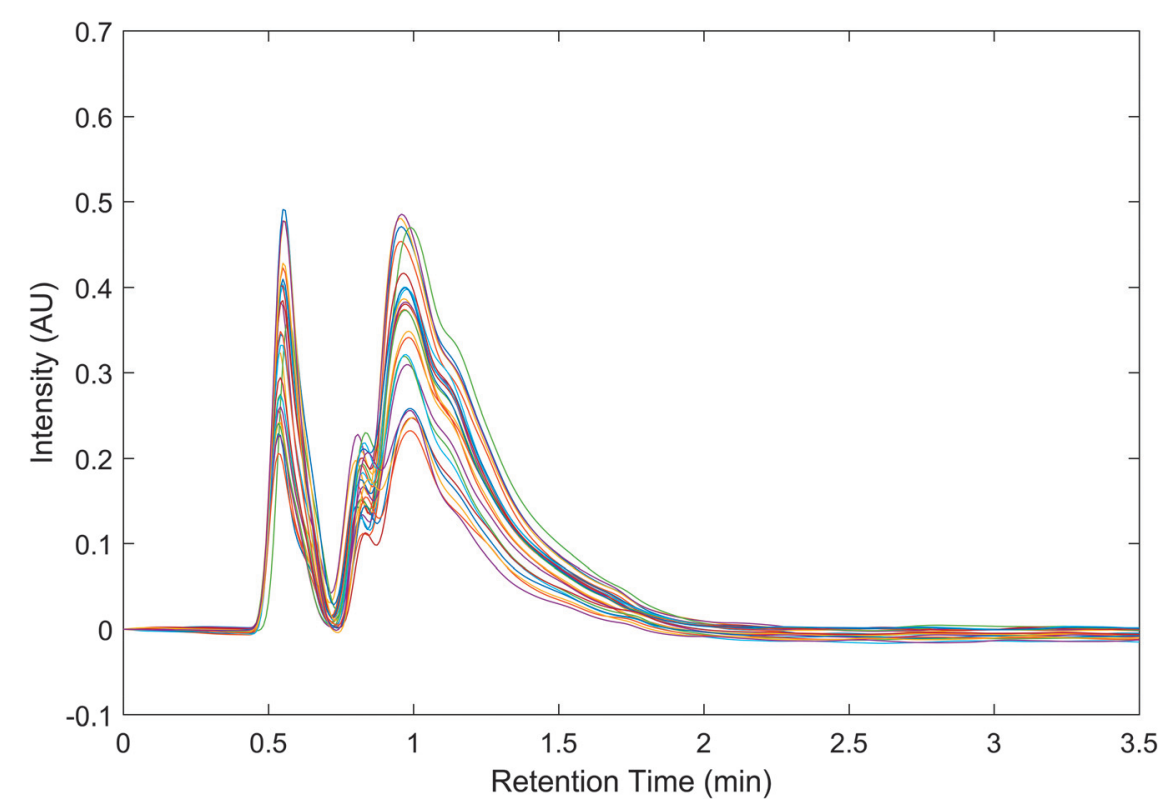

Figure 2. The shifts of peaks in retention time (standard samples, $\lambda=210 \mathrm{~nm}$ ) 

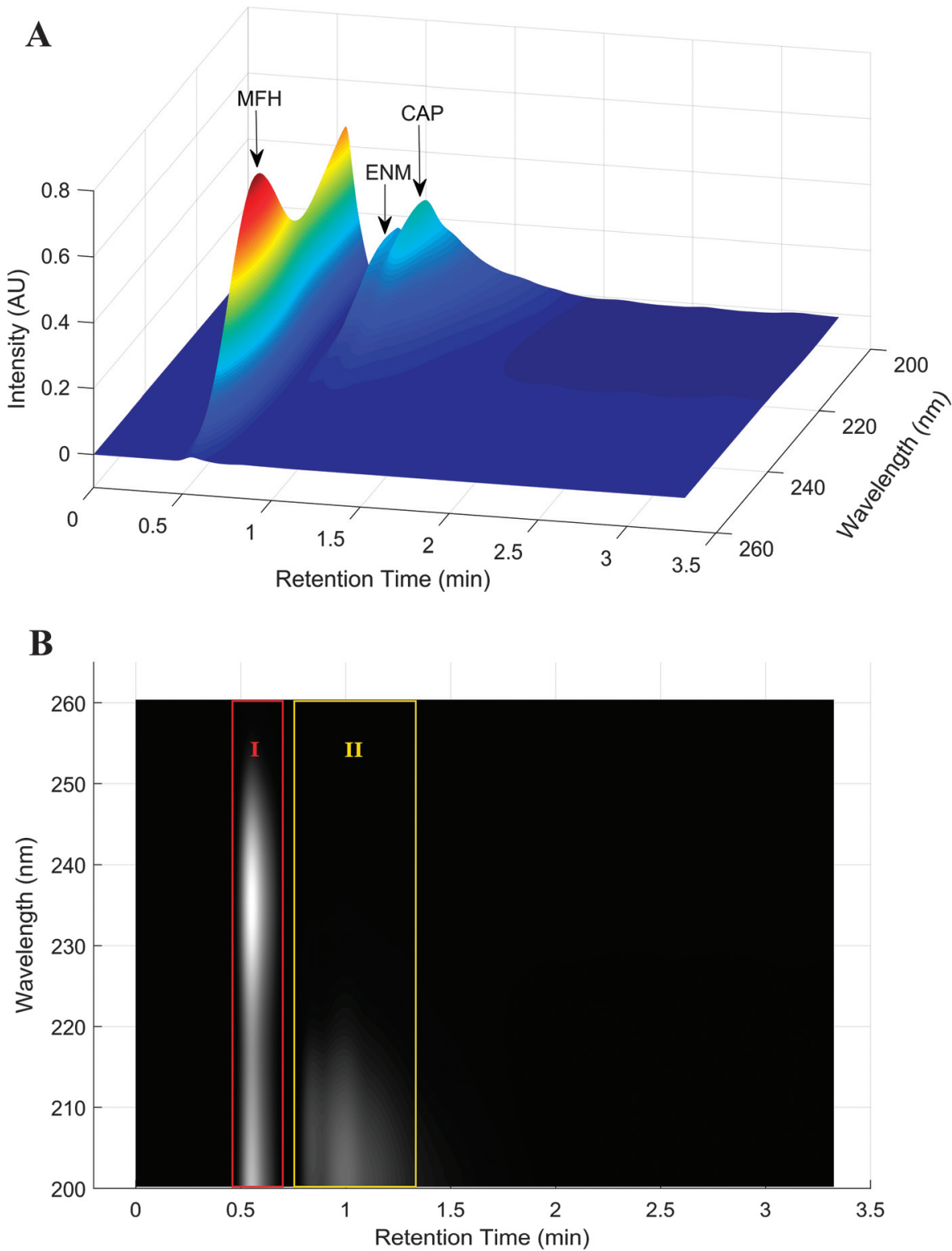

Figure 3. The HPLC-PAD spectrum of one sample: (A) 3D spectrum and (B) grayscale image

3.3. Validation of the Models and Methods. The statistical parameters of obtained models and the validation indexes of the proposed methods are listed in Table 2. As can be seen from Table 2, the correlation coefficients $(R)$, the adjusted $R\left(R_{\text {adj }}\right)$, and the cross-validation $\left(R_{\mathrm{LOO}-\mathrm{cv}}\right)$ of the models established by TMs are more than 0.98 , and this shows a subtle superiority of TMs over MCR-ALS. These results indicate that the quantitative relationship between the

Table 2. The performance of the obtained models and the analytical results of real tablets

\begin{tabular}{|c|c|c|c|c|c|c|}
\hline \multirow[t]{2}{*}{ ITEM } & \multicolumn{2}{|c|}{ MFH } & \multicolumn{2}{|c|}{ ENM } & \multicolumn{2}{|c|}{ CAP } \\
\hline & TM & MCR-ALS & TM & MCR-ALS & $\mathrm{TM}$ & $\overline{\text { MCR-ALS }}$ \\
\hline Correlation coefficient $(R)$ & 0.994 & 0.972 & 0.995 & 0.964 & 0.997 & 0.982 \\
\hline Adjusted correlation coefficient $\left(R_{\mathrm{adj}}\right)$ & 0.992 & 0.970 & 0.993 & 0.962 & 0.996 & 0.981 \\
\hline Correlation coefficient of LOO $\left(R_{\mathrm{LOO}-\mathrm{CV}}\right)$ & 0.990 & 0.968 & 0.988 & 0.957 & 0.993 & 0.977 \\
\hline Root mean square error (RMSE) & 4.599 & 9.372 & 5.715 & 13.095 & 16.16 & 37.623 \\
\hline Root mean square error cross validation $\left(\mathrm{RMSE}_{\mathrm{CV}}\right)$ & 5.172 & 10.116 & 7.399 & 14.318 & 21.62 & 42.110 \\
\hline Correlation coefficient of prediction $\left(R_{\mathrm{p}}\right)$ & 0.993 & 0.997 & 0.974 & 0.877 & 0.988 & 0.920 \\
\hline Root mean square error of prediction $\left(\mathrm{RMSE}_{\mathrm{p}}\right)$ & 10.08 & 2.946 & 12.31 & 20.190 & 33.15 & 56.037 \\
\hline$F$-Test value & 383 & 276 & 333 & 212 & 380 & 433 \\
\hline$p$-Value & $1.15 \times 10^{-13}$ & $1.65 \times 10^{-11}$ & $5.94 \times 10^{-13}$ & $1.21 \times 10^{-10}$ & $4.30 \times 10^{-12}$ & $5.18 \times 10^{-13}$ \\
\hline Recovery $(\% \pm R S D)$ & $100.1 \pm 1.7$ & $92.9 \pm 2.1$ & $95.40 \pm 5.4$ & $67.6 \pm 3.9$ & $105.3 \pm 5.7$ & $88.5 \pm 2.5$ \\
\hline \multicolumn{7}{|l|}{ Precisions $(R S D(\%, n=3))$} \\
\hline Intra-day & 2.128 & 1.73 & 3.963 & 0.386 & 1.702 & 2.614 \\
\hline Inter-day & 2.852 & 1.332 & 5.424 & 6.572 & 2.003 & 1.279 \\
\hline \multicolumn{7}{|l|}{ Linearity $(n=25)$} \\
\hline Test range $\left(\mu \mathrm{g} \mathrm{mL}^{-1}\right)$ & $100-240$ & $100-240$ & $140-300$ & $100-240$ & $500-1200$ & $100-240$ \\
\hline Slope & 0.9915 & 0.953 & 0.9931 & 0.879 & 0.9947 & 0.947 \\
\hline Content (mg) & 399.6 & 477.6 & 0.28 & 0.19 & 0.16 & 0.14 \\
\hline
\end{tabular}



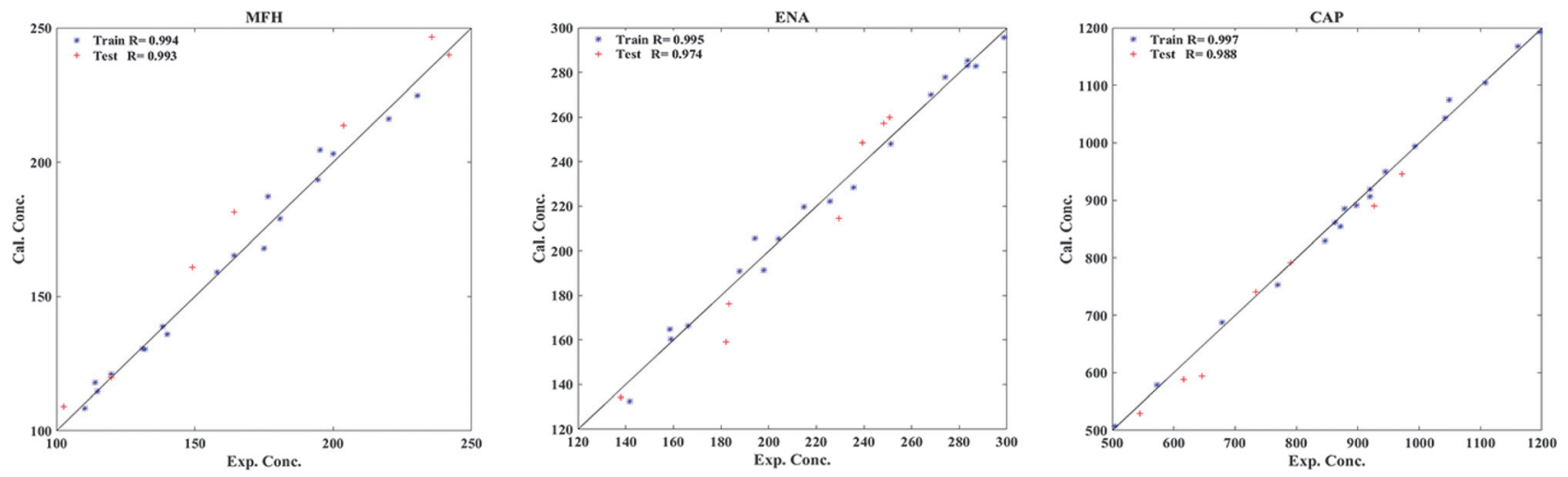

Figure 4. The experimental vs. calculated conc. $(\mu \mathrm{g} / \mathrm{mL})$ with TM model for calibration and prediction of MFH, ENM, and CAP

concentrations and selected TMs is reliable. $F$-test values were yielded within the range of hundreds and in line with the rule saying that the higher this value is, the more significant the model is, and also the remarkably obtained low $p$-values meet the statistical requirements. The RMSE and RMSE $\mathrm{cv}_{\mathrm{cv}}$ for TMs are lower for the overlapped components comparing with the ones for MCR-ALS, which means TMs significantly handle this issue.

The accuracy of the method was estimated through a recovery test, which was determined by a standard addition method [31]. As can be seen from Table 2, the recoveries (\%) for $\mathrm{MFH}$, ENM, and CAP calculated from Eq. 10 are 100.1 \pm 1.7 , $95.4 \pm 5.4$, and $105.3 \pm 5.7$ and $92.9 \pm 2.1,67.6 \pm 3.9$, and $88.5 \pm 2.5$ for TMS and MCR-ALS, respectively. The precision (RSD\%) of intra- and inter-day are less than 6.5 for both methods. The good slope values support the sensitivity of the models, especially for TMs. Calibration and external validation were carried out by splitting the dataset into training (18) and test (7) sets. The relationship between the experimental (Exp.) concentrations and the calculated (Cal.) concentrations of the three target components is illustrated in Figure 4 for the model established by Tchebichef moments. These results indicate that TM method outperforms the MCR-ALS approach.

3.4. Real Sample Analysis. For the real tablets including MFH, ENM, and CAP, the quantitative analysis of the three target components was performed by means of the proposed method and the MCR-ALS method, and the obtained results are also listed in Table 2. For the proposed method, the average concentrations of MFH, ENM, and CAP are $87.7 \pm 1.9$, $12.5 \pm 2.5$, and $31.3 \pm 3.1 \mu \mathrm{g} / \mathrm{mL}$, respectively. Accordingly, the contents $(\mathrm{mg})$ of the three target components in one tablet are $399.6(\mathrm{MFH}), 0.28(\mathrm{ENM})$, and 0.16 (CAP).

\section{Conclusion}

In this work, we successfully applied different methods on 3D spectra of the HPLC-PAD to establish the linear quantitative models for the rapid analysis of metformin hydrochloride $(\mathrm{MFH})$, enalapril maleate (ENM), and captopril (CAP) in tablets. Owing to the advantages of discrete orthogonality, inherent invariance, and multi-resolution properties, as well as its unit weight function, the Tchebichef moments method coupled to stepwise regression transcends MCR-ALS, and it could reduce the analytical time, simplify the experimental operations, predicting the determination of the investigated drugs in tablets, and improve the intended analytical accuracy. Therefore, Tchebichef image moments method could be a potential technology for the routine analytical and quality control of the tablets including $\mathrm{MFH}, \mathrm{ENM}$, and CAP.
Acknowledgement. The authors use this chance to thank the National Natural Science Foundation Committee of the P.R. China (Grant No. 21275067) for its financial support during this research work.

\section{References}

1. Organization, W. H. Definition and diagnosis of diabetes mellitus and intermediate hyperglycaemia: report of a WH $\mathbf{2 0 0 6}$.

2. (a) Shenfield, G. Aust. Prescr. 2013, 36, 38-39; (b) Nathan, D.; Buse, J. B.; Davidson, M. B.; Ferrannini, E.; Holman, R. R.; Sherwin, R.; Zinman, B. Diabetologia 2009, 52, 17-30; (c) Campbell, I. W. Arq. Bras. Endocrinol. Metabol. 2000, 44, 121-124.

3. Klepser, T. B.; Kelly, M. W. Am. J. Health-Syst. Pharm. 1997, 54, 893-903.

4. (a) Garber, A. J.; Duncan, T. G.; Goodman, A. M.; Mills, D. J.; Rohlf, J. L. Am. J. Med. 1997, 103, 491-497; (b) Hu, L.-D.; Liu, Y.; Tang, X.; Zhang, Q. Eur. J. Pharm. Biopharm. 2006, 64, 185-192.

5. Mogensen, C. E. Diabetologia 1999, 42, 263-285

6. (a) Ahmed, S.; Atia, N. N.; Mohamed, N. A. Talanta 2011, 84, 666-672; (b) Yardimci, C.; Ozaltin, N.; Gurlek, A. Talanta 2007, 72, 1416-1422; (c) Shafi, N.; Siddiqui, F. A.; Sultana, N.; Arayne, M. S. J. Liq. Chromatogr Relat. Technol. 2015, 38, 1466-1473.

7. Lokesh, B.; Naidu, S. R. JASA 2007, 2, 34-37.

8. Heel, R. C.; Brogden, R. N.; Speight, T. M.; Avery, G. S. Drugs 1980 20, 409-452.

9. (a) Khamanga, S. M.; Walker, R. B. Dissolut. Technol. 2012, 19, 42-51; (b) Davies, R. O.; Gomez, H. J.; Irvin, J. D.; Walker, J. F. Br. J. Clin. Pharmacol. 1984, 18, S215-S229.

10. (a) Peraman, R.; Gowra, C. S.; Reddy, Y. P.; Peruru, K. K. Chromatographia 2013, 76, 1153-1162; (b) Gite, S.; Patravale, V. J. Chromatogr. Sci. 2015, 53 1654-1662; (c) Chhetri, H. P.; Thapa, P.; Van Schepdael, A. Saudi Pharm. J. 2014 $22,483-487$.

11. Chen, J.; Li, B. Q.; Zhai, H. L.; Lu, W. J.; Zhang, X. Y. J. Chromatogr. A 2014, 1352, 55-61.

12. (a) Arayne, M. S.; Sultana, N.; Zuberi, M. H.; Siddiqui, F. A.; Haroon, U. Med. Chem. Res. 2013, 22, 5717-5722; (b) Cumar, R. P.; Vasudevan, M.; Deecaraman, A. Rasayan J. Chem 2012, 5, 137-141.

13. (a) Panigrahy, U. P.; Reddy, A. S. K. Orient. J. Chem. 2015, 31, 1489-1507; (b) Monakhova, Y. B.; Mushtakova, S. P.; Kolesnikova, S. S.; Astakhov, S. A. Anal. Bioanal. Chem. 2010, 397, 1297-1306.

14. (a) Mukundan, R.; Ong, S. H.; Lee, P. A. IEEE Trans. Image Process. 2001, 10, 1357-1364; (b) Dai, X. B.; Shu, H. Z.; Luo, L. M.; Han, G. N.; Coatrieux, J. L. Pattern Recognit. Lett. 2010, 43, 1152-1164.

15. Zhu, H.; Shu, H.; Zhou, J.; Luo, L.; Coatrieux, J. L. Pattern Recognit. Lett. 2007, 28, 1688-1704.

16. Yang, P. C. Formulation of invariants for discrete orthogonal moments and image classification University of Malaya 2013.

17. Chen, J.; Li, B. Q.; Xu, M. L.; Wang, X.; Jing, Y. H.; Zhai, H. L. Talanta 2016, 161, 99-104.

18. Xu, M. L.; Li, B. Q.; Wang, X.; Chen, J.; Zhai, H. L. J. Sep. Sci. 2016 39, 3054-3061.

19. (a) Li, B. Q.; Chen, J.; Xu, M. L.; Wang, X.; Zhai, H. L Chemom. Intell. Lab. Syst. 2016, 156, 128-136; (b) Li, B. Q.; Xu, M. L.; Wang, X.; Zhai, H. L.; Chen, J.; Liu, J. J. Food Chem. 2017, 216, 52-59; (c) De Luca, S.; Ciotoli, E.; Biancolillo, A.; Bucci, R.; Magrì, A. D.; Marini, F. Environ. Sci. Pollut. Res. 2018.

20. Navea, S.; de Juan, A.; Tauler, R. Anal. Chem. 2003, 75, 5592-5601.

21. Garrido, M.; Rius, F. X.; Larrechi, M. S. Anal. Bioanal. Chem. 2008, 390, 2059-2066

22. (a) Sinanian, M. M.; Cook, D. W.; Rutan, S. C.; Wijesinghe, D. S. Anal. Chem. 2016, 88, 11092-11099; (b) Azimi, F.; Fatemi, M. H. RSC Adv. 2016, 6, 111197-111209.

23. de Juan, A.; Rutan, S. C.; Tauler, R.; Massart, D. L. Chemometrics Intell. Lab. Syst. 1998, 40, 19-32. 
24. Shu, H. Z.; Zhang, H.; Chen, B. J.; Haigron, P.; Luo, L. M. IEEE Trans. Image Process. 2010, 19, 3171-3180.

25. Li, B. Q.; Chen, J.; Li, J. J.; Wang, X.; Zhai, H. L. Analyst 2015, 140 $630-636$.

26. Mukundan, R.; Ong, S. H.; Lee, P. A. IEEE Trans. Image Process. 2001, 10, 1357-64.

27. See, K. W.; Loke, K. S.; Lee, P. A.; Loe, K. F. Appl. Math. Comput. 2007, 193, 346-359.
28. Costa, P.; Sousa Lobo, J. M. Eur. J. Pharm. Sci. 2001, 13, 123-133.

29. (a) Taverniers, I.; De Loose, M.; Van Bockstaele, E. Trac-Trends Anal. Chem. 2004, 23, 535-552; (b) Huber, L. Validation of analytical methods Agilent technologies, 2010, 13-28.

30. Shu, H. Z.; Zhou, J.; Han, G. N.; Luo, L. M.; Coatrieux, J. L. Pattern Recognit. 2007, 40, 670-680.

31. Hussain, J. N.; Sumanoja, S. L. Int. J. Pharm., Chem. Biol. Sci. 2013, $3,546-553$. 